\title{
Modeling of Transient Nonlinear Phenomena using a Modified Logistic Equation
}

\author{
Tomáš Stejskal ${ }^{1, *}$, Jozef Svetlík ${ }^{1}$, Peter Demeč ${ }^{1}$ and Luboslava Šidlovská ${ }^{1}$ \\ 'Technical University of Košice, Faculty of Mechanical Engineering, Department of Production Technique, Letná 9, 04000 Košice, Slovakia
}

\begin{abstract}
The paper deals with modelling of nonlinear transient responses that occur in the technical and natural objects. In the field of non-linear phenomena are generated structures that have regular and chaotic nature. As a modelling tool, we used a modification of the logistic equation. Search and display of various structures was achieved when using parameter iterations of modified logistic equation. An iterative method in this case appears to be a very effective tool. The novelty of the method is in creating regular and chaotic structures in the time development of nonlinear functions.
\end{abstract}

Keywords: nonlinear dynamic, logistic map, logistic function, chaos.

\section{Introduction}

Transient responses are present in all mechanisms. Transient response is generally defined as a physical change between two stabilzed states. In this article we will deal with special cases of transients that can be defined as a physical change between steady state and unstable (divergent) state. It is on the border of these states where processes, that can be described as chaotic and bifurcation processes, are taking place.

There is no physical system where non-linearity is not found. The question is to what extent the non-linearity of the system occurs during given conditions. Chaotic manifestation of nonlinearity appears in a measurable way on physical objects. Furthermore, it appears in the calculation schemes in analytical solution system. Finally, it also appears in the modeling of some recurrent relations.

\subsection{Nonlinear models of physical objects}

The first discovery of chaotic behaviour of nonlinear system was a BelousovZhabotinsky reaction published in 1958 by Belousov as citric acid oxidation of bromate ions in the presence of cerium ions. The reaction was interesting in its visual effect where the system color was oscillatory changing between yellow and colorless solution [1].

Another example is nonlinearity occurrence in the resonance space of the oscillating system. This behavior has been revealed by analysis of seismic activity in the Lake Baikal region. In the Baikal region there is a extension of the Earth's crust and the formation of rift [2]. This process can be modeled by bifurcation in nonlinear resonance of hysteresis curve (Fig. 1)

It is a model of behavior that often occurs in the mechanical and electrical systems. In case of strong nonlinearity there is no analytical solution of the nonlinear equations. The behavior of the system can only be addressed by modeling. Nonlinear behavior of the mechanical system is reflected for example in the vibration 
of rolling mill rolls [3]. In this case, the nonlinearity of cylinders' movement was analytically solved by two perpendicular to each other planes (Fig. 2).

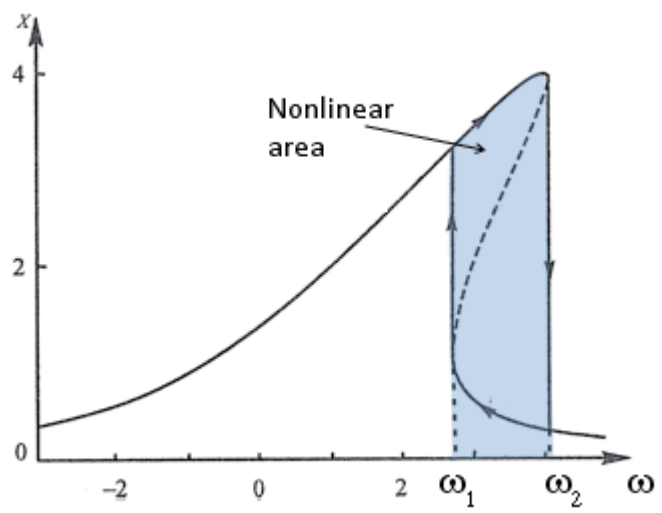

Fig. 1: Nonlinear resonance hysteresis. Arrows show direction of motion along the resonance curve in the case of slow change in excitation frequency.

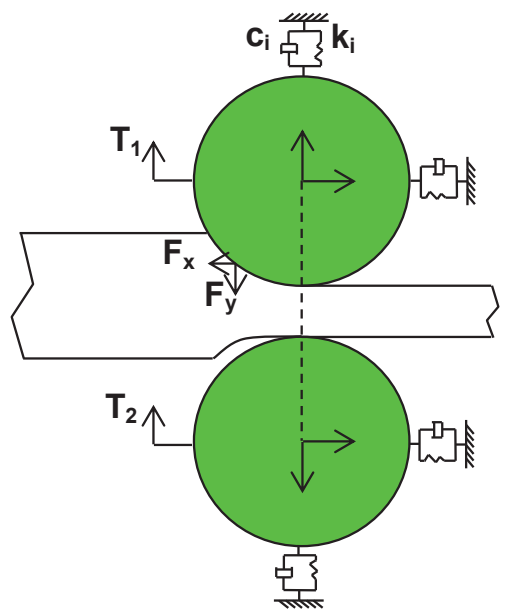

Fig. 2: The vertical-horizontal coupling dynamic model of rolling mill rolls.

System instability is created also by the splintery machining. The negative consequences are deterioration of the surface and rapid tool wear. This is an area of self-excited tool oscillations. Near the border between stability and instability is an area of bistability (Fig. 3). Near the stability border are arising unstable periodic cycles that due to outside interference may develop into stable periodic oscillation cycles [4], [5].

\subsection{Nonlinearity of calculation procedures}

Nonlinearity is a frequent calculation phenomenon when describing dynamic structures. A typical example is the investigation of the stability of a

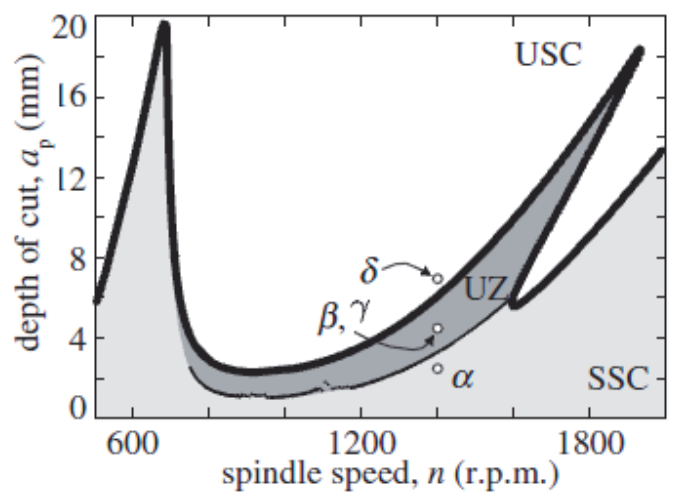

Fig. 3: The calculated lower boundary of the unsafe (or bistable) zone (UZ), which is coloured dark grey embedded in the grey region of linearly stable stationary cutting.

bar strained for buckling. This task was first solved by Euler (1744). Differential equations describing the phenomenon are non-linear. Solution for continuous model leads to a smooth curve in phase space. The discrete model is giving even more interesting results [6]. In this case is considered a bar that consists of several segments. The resulting stability limit in phase space has a complex and simultaneously typical fractal nature (Fig. 4).

(a)
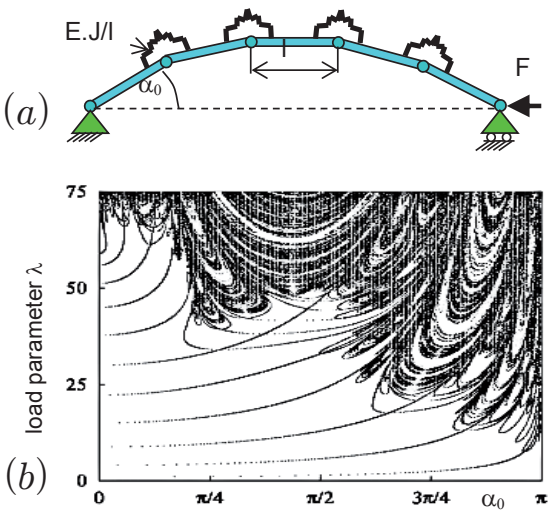

Fig. 4: (a) The mechanical discretization of on endloaded rod, (b) Global bifurcation diagram of elastic linkages for the $N=12$-element chain.

Fractals are the result of the structurally simple pendulum system too. For example, the double pendulum at a certain initial energy has no longer analytically defined trajectory [7], [8]. Poincaré map has a fractal nature (Fig. 5).

\subsection{Nonlinearity of recurrent relations}

The third investigation area of chaotic systems is creation of discrete mathematical model while 


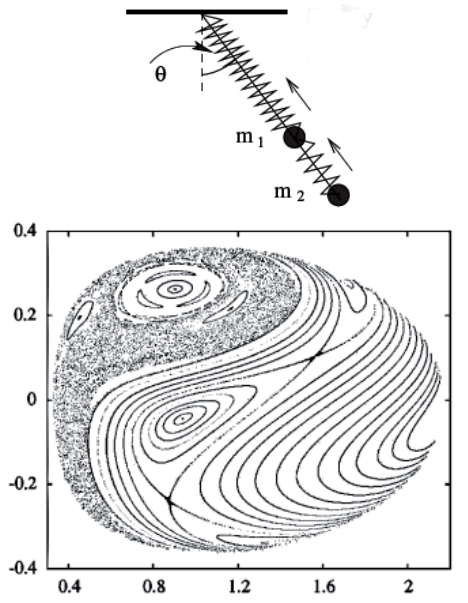

Fig. 5: The Poincaré sections for two rigid spring pendula.

using the recurrent relations. The most famous model is the logistic function (1). This general formulation is the basis for the formation of simple ecological discrete models of population growth. The simplest example of a discrete nonlinear model is the logistic map, for which $x$ changes from time $n$ to time $n+1$ according to:

$$
x_{n+1}=r\left(x_{n}-x_{n}^{2}\right)
$$

Where $r$ is a control parameter which is describing the growth rate of population. Recurrent equation exhibits chaotic behavior only for certain values of the control parameter $r$.

Convergence ratio of dubling the number of bifurcation nodes has universal character [9]. When parameter $r=3,569945672$, number of bifurcation nods is infinite and the system behaves chaotically. Such bifurcation value of the parameter $r$ is called accumulation point. Character behavior of the system determines the bifurcation diagram (Fig. 6).

Bifurcation diagram has the characteristics of fractal. The principal of the diagram interpretation is based on analysis of control parameter $r$ changes. Nonlinear systems can have more parameters and each parameter changes the conditions for development of the system. In our case, parameter $r$ represents the boundaries of system stability. Each nonlinear system on the border of stability recognized the following areas (Fig. 6):

$\checkmark$ Stable region - one cycle, analytical solution is possible.

$\checkmark$ Bifurcation region - regular cycles, analytical solution is possible.

$\checkmark$ Chaotic region - irregular cycles, analytical solution is not possible.

$\checkmark$ Unstable region - closing of cycles, the system diverges.

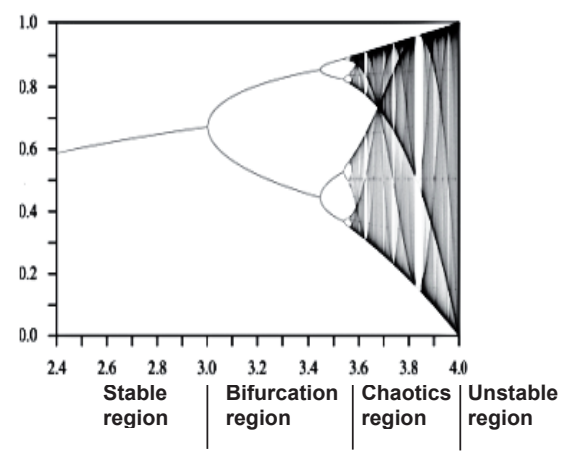

Fig. 6: Bifurcation diagram of the logistic map.

By the multi parametric systems similar regions occur [10]. When parameters of recurrent equation are selected properly, the system seeks to stabilize at one point in which it oscillates. It's a stable region. To deflect the oscillations continues changing of parameters is needed. In the border area, the system enters the bifurcation area where regular structure are being formed and the system oscillates at several points. With further parameter changes the system will either diverge, or get into the area of chaotic structures. By chaotic structures the system creates oscillations in non-recurrent points. After further changes the system begins to diverge.

\section{Modification of logistic equation}

Logistic map is displayed relationship $x_{n}-x_{n-1}$ and the logistics equation is always a parabolic course (Fig. 7). In the time domain disordered chaotic structure occurs, while connecting lines between steps are not shown. With any change of parameter $r$ there in no change of parabola.

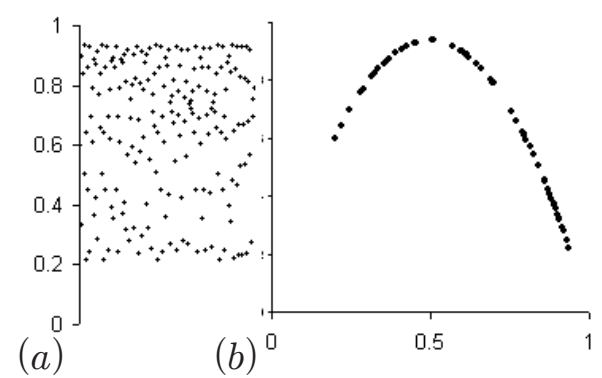

Fig. 7: (a) The time domain of logistic equation, (b) The logistic map (parameter $r=3.75$ ). 
The functional relationship is given as

$x_{n+1}=f\left(x_{n}\right)$

Modification of the relationship consists in adding a new recurrent step that takes into account the state in the previous step.

$$
x_{n+1}=f\left(x_{n}, x_{n-1}\right)
$$

To generate changes additional parameters may be used. The general formulation of the modified equation is as follows:

$$
x_{n+1}=r\left(x_{n} \pm t \cdot x_{n-1}-v \cdot x_{n}^{g}\right)
$$

Each element of the modified equation plays a role in formation of the resulting graphic curve in the logistic map and the time domain. The most feasible method for generating structures in time domain is the use of parameters iteration. This makes it possible to generate a two dimensional plot on the boundary of system stability. The procedure is similar to the creation of the bifurcation diagrams of the primary logistic equation.

Parameters $t, r$ allow to modify the function to structures that are regular in the time domain (belong to the bifurcation region) and they are on the borderline of stability and divergence.

The parameters $\mathrm{v}, \mathrm{g}$ enable modification of the function to structures which are chaotic in the time domain and the logistic map too. These structures are on the edge of chaos and divergence.

\section{Generation of regular structures - Experimental part}

The modification consists in subtracting or adding element $x_{n-1}$ in one step. If the parameter is $v=0$ then only linear recurrent equation is formed:

$$
x_{n+1}=r\left(x_{n}-t \cdot x_{n-1}\right)
$$

or

$$
x_{n+1}=r\left(x_{n}+t \cdot x_{n-1}\right)
$$

It can be verified, that for any combination of input parameters the equation (6) has only divergent solution. Despite that, the equation (5) is oscillating when appropriate parameters are

chosen. This oscillation is the base for the structures creation. Mutual parameter $\mathrm{r}$, t ration defines the boundaries of convergence or divergence of recurrent equation (5). In case $r=\frac{1}{t}$ the equation (5) is exactly on the border of convergence and divergence (Fig. 8b).

\subsection{Display of structures}

The structure display of nonlinear states is as follows. In the all figures on the left are shown still 5000 steps of element. It is function time domain without connecting lines between steps. In the second part of the figures on the right is the logistic map of the same function. There are still displayed 1000 steps (Fig. 8).
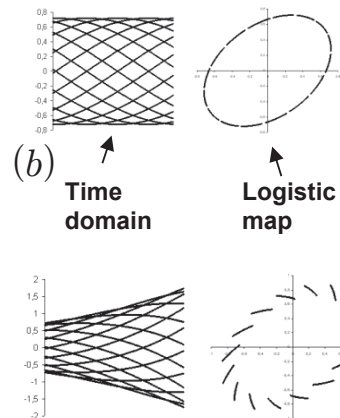

(c)

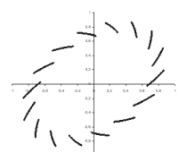

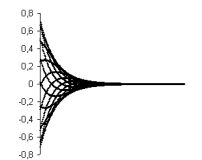

(a)

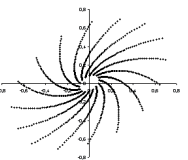

政




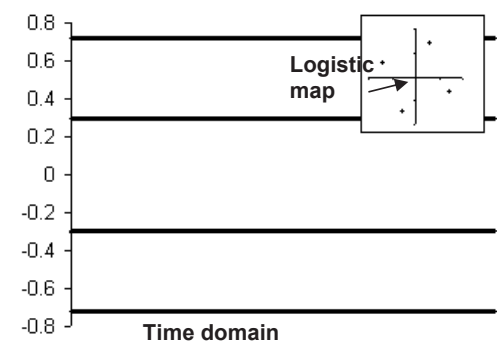

Fig. 9: The four steps in one cycle by parameters: $x_{0}=0.3 \quad x_{1}=0.72$ $r=0.000001 \quad t=1000000$.

the resulting map can still be seen whether it is necessary to increase or decrease the parameter to achieve the threshold. On the basis of experiments, we assume that this is a general principle guiding the behavior of nonlinear systems. The proposed iterative method is fast and cannot be qualified as an accidental type of method as a trial - error method. But it is not analytical method. However, neither is the generation of chaotic waveforms in any system. Table 1 displays the parameter value $r$ in integer steps ratios. The tendency of $r$ parameter growth based on actions taken expresses the convergence to number two. For $r \geq 2$ the function diverges. Values in the table have been gained by $r$ parameter iteration and based on this we have derived generally valid relationship between exact steps $n$ and parameter $r$.

Table 1: The values of the parameter $r$ in the whole-number proportion of the cycle.

\begin{tabular}{|l|l|c|}
\hline $\begin{array}{l}\text { Number of } \\
\text { steps in cycle }\end{array}$ & Mathematical expresion \\
\hline 4 & 0 & \\
\hline 5 & 0.618034 & $\frac{\sqrt{5}-1}{2}$ \\
\hline 6 & 1 & \\
\hline 7 & 1.24698 & \\
\hline 8 & 1.414214 & $\sqrt{2}$ \\
\hline 9 & 1.532089 & \\
\hline 10 & 1.618034 & $\sqrt{5}+1$ \\
\hline 11 & 1.682508 & \\
\hline 12 & 1.732051 & $\sqrt{3}$ \\
\hline 13 & 1.770912 & \\
\hline 14 & 1.801938 & \\
\hline 15 & 1.827091 & \\
\hline
\end{tabular}

From a geometric interpretation of a regular polygon we have derived an analytic function (7) which expresses relation between the number of steps $n$ in the cycle and parameter $r$ (Fig. 10).

$$
r=\frac{2 \cdot \cos \frac{2 \pi}{n} \cdot \operatorname{tg} \frac{\pi}{n}}{\sin \frac{2 \pi}{n}-\cos \frac{2 \pi}{n} \cdot \operatorname{tg} \frac{\pi}{n}}
$$

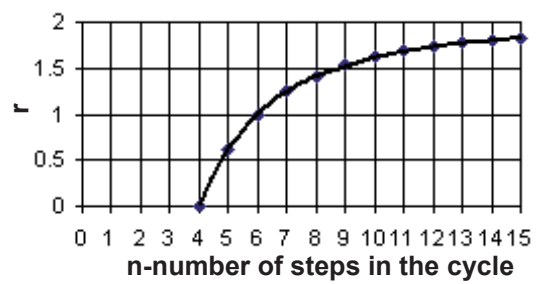

Fig. 10: The change of parameter $r$ depending on the number of steps in the cycle.

\subsection{Examples of regular structures - bifurcations region}

By not integer proportion of step the parameter $r$ change, generates a range of regularly structures (Fig. 11), (Fig. 12), (Fig. 13). Initial conditions are unchanged. But change in the structure, can also be achieved by changing the initial conditions.

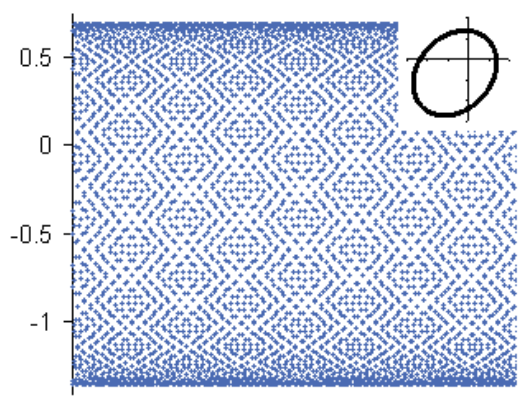

Fig. 11: Parameters: $x_{0}=0.3 \quad x_{l}=0.6 \quad r=0.5 \quad t=2$.

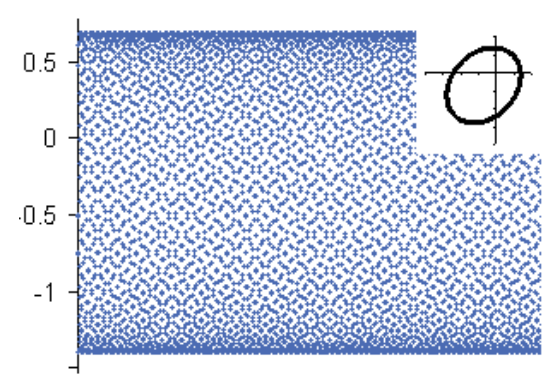

Fig. 12: Parameters: $x_{0}=0.3 \quad x_{1}=0.6 \quad r=0.525 \quad t=1.904761905$. 


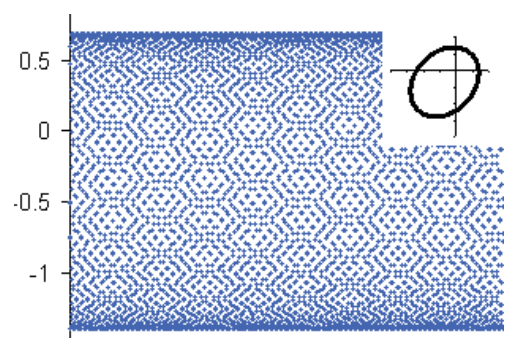

Fig. 13: Parameters: $x_{0}=0.3 \quad x_{1}=0.6 \quad r=0.52533 \quad t=1.903565378$.

\section{Generation of chaotic structures}

The general equation (4) can be simplified to the form of the exponential recurrent equation. Parameter $v=1$ and $t=0$.

$$
x_{n+1}=r\left(x_{n}-x_{n}^{g}\right)
$$

The role of the exponent $g$ is to enable the equation to get to the border of chaos. In this sense, the chaos as a transition state between function convergence to the finite integer figures and divergence. For $g=2$ it is a classic logistics equation. Chaotic waveforms are generated by the choice of parameters $g=3, g=4$ and higher values (Fig. 14). Special structures even in area of the logistic map can be made with the equation (4) (Fig. 15), (Fig. 16) and (Fig. 17).

\subsection{Examples of chaotic structures}

Although the structures in various parameter changes are different, the scenario process from regularity to the divergence is same. The size of the necessary change is defined by iteration of parameter. This change cannot be calculated. It's a chaotic system where every little thing can change the whole state. For example, if we maintain all the specified parameters and only change the calculation accuracy of the one decimal point, it changes the whole structure. The desired state can be achieved, but for other parameter values.

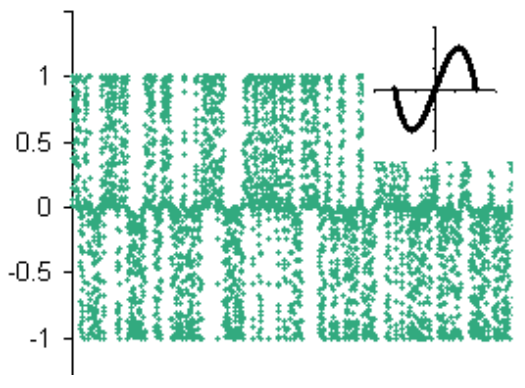

Fig. 14: Parameters: $x_{0}=0.6 r=2.6 g=3$.

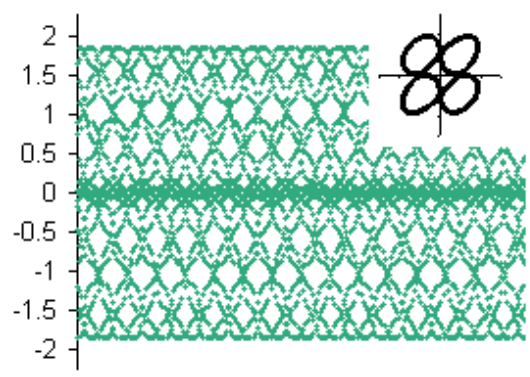

Fig. 15: Parameters: $x_{0}=0.1 \quad x_{1}=0.45684 \quad r=1.2 \quad t=0.8333333333$ $g=1 / 3$.

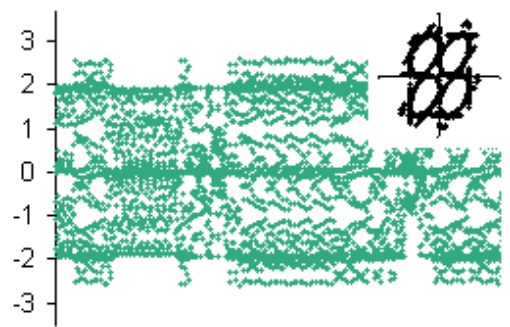

Fig. 16: Parameters: $x_{0}=0.1 \quad x_{1}=0.3 \quad r=1.2 \quad t=0.833333333 \quad g=1 / 5$

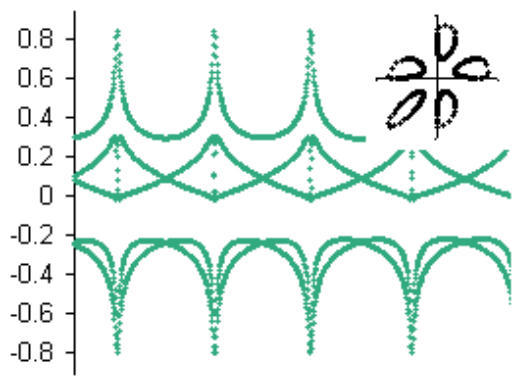

Fig. 17: Parameters: $x_{0}=0.1 \quad x_{1}=0.3 \quad r=0.618034 \quad t=1.618033959$ $g=4$.

\section{Conclusion}

Main contribution of this article is to point out that nonlinear systems on the border of stability report under the universal principle that can be modeled by modified logistic equation. It's just a question of picturing structures so that these principles of behavior are visible. There are very interested structures formed in the time domain of functions development. This display brings novelty to the chaos theory.

Based on the experience so far we affirm that every nonlinear system before it starts divergence passes through the zone bifurcation and consequently through the zone of chaotic behavior. Although in practice this fact is not clearly observed, it is due 
to the fact that it is a quick process with the low number of occurrence. From the technical systems we rarely require to have unstable behavior. Moreover, the real technical objects are affected by the noise of random processes that effectively overlap chaotic manifestations. Moreover, the real technical objects are affected by the noise of random processes that effectively overlap chaotic manifestations.

The shapes of maps that arise in the transition regions have their universal nature in the time domain. In the bifurcations region they are the regular geometric shapes. In the chaos region they are the fractal formations that are by the gradual change of parameter alternating with regular formations. The most interesting forms, of course, occur at the limits, close to instability (divergence) regardless of which parameter is being changed by small additional values. In the last phase on the border of stability structures are alternating with bands of divergence. Finally, any change of parameters formes then no structure.

\section{Acknowledgments \\ Contribution was created under grant projects KEGA 039TUKE-4/2016 and VEGA 1/0124/15.}

\section{References and Notes}

[1] VANAG, Vladimir K.; EPSTEIN, Irving R. Pattern formation in a tunable medium: The Belousov-Zhabotinsky reaction in an aerosol OT microemulsion. Physical review letters, 2001, 87.22: 228301.

[2] KLYUCHEVSKII, Anatoly V.; KHLEBOPROS, Rem G. Coupled large earthquakes in the Baikal rift system: Response to bifurcations in nonlinear resonance hysteresis. Geoscience Frontiers, 2013, 4.6: 709-716.

[3] HOU, Dongxiao; PENG, Rongrong; LIU, Haoran. Analysis of vertical-horizontal coupling vibration characteristics of rolling mill rolls based on strip dynamic deformation process. Shock and Vibration, 2014, 2014.

[4] DOMBOVARI, Zoltan; STEPAN, Gabor. On the bistable zone of milling processes. Phil. Trans. R. Soc. A, 2015, 373.2051: 20140409.

[5] DOMBOVARI, Zoltan; WILSON, R. Eddie; STEPAN, Gabor. Estimates of the bistable region in metal cutting. In: Proceedings of the Royal Society of London A: Mathematical, Physical and Engineering Sciences. The Royal Society, 2008. p. 3255-3271.

[6] DOMOKOS, G.; HOLMES, P. Euler's problem, Euler's method, and the standard map; or, the discrete charm of buckling.
Journal of Nonlinear Science, 1993, 3.1: 109-151.

[7] SHINBROT, Troy, et al. Chaos in a double pendulum. Am. J. Phys, 1992, 60.6: 491-499.

[8] SZUMINSKI, Wojciech Dynamics of multiple pendula without gravity: Chaotic Modeling and Simulation - CMSIM -2014, no. 1, pp. 57-67

[9] FEIGENBAUM, Mitchell J. Quantitative universality for a class of nonlinear transformations. Journal of statistical physics, 1978, 19.1: 25-52.

[10] ČAČKO, Peter; KRENICKÝ,Tibor. Impact of Lubrication Interval to Operating Status of Bearing. In: Applied Mechanics and Materials. Trans Tech Publications, 2014. p. 151-158.

\section{Biographical notes}

Tomás Stejskal, Ing. PhD., (born in 1963). Is an assistant lecturer at the Department of Production Technique at the Faculty of Mechanical Engineering, Technical University of Košice. He graduated at the Department of Machine Tools and Equipment, which was a predecessor of the above-mentioned Department in 1988. The PhD.-degree he obtained with the dissertation thesis target on the issue of technical diagnosing and failure states of production machinery. His research activities are focused on the machine heath diagnosis, signal analysis and measurement of technical parameters of production machinery.

Jozef Svetlik, doc. Ing. PhD., (born in 1977) associate professor at the Department of manufacturing engineering at the Faculty of Mechanical Engineering in TU Košice (SjF, TUKE). Graduade TUKE SjF 2000, scientific degree of Doctor of philosophy (PhD.) defended at Technical University of Košice in 2004. Internal PhD. student SjF TUKE (2000-2003), academic degree of associate professor (Doc.) defended at Technical University of Košice in 2012. Winner of "Siemens Excellence Award 2004" for the disertation thesis. The holder of an honorary recognition of the International Engineering Fair 2003 in Nitra Agrokomplex for "inteligent modular assembly system". Team member "SjF Tuke robotics", which is several times European champion and world champion from 2010 in robotic soccer. Author of a utility model "Rotary module for modular construction machinery" and patent "contactless transmission of electrical power for modular machines". Research interests of construction machinery and modular robotics. Member of the editorial board of "Journal of Applied Statistics". Co-autor of the script "Modules for the construction of production machinery and robotics - Technical equipment for automated workplaces: inter-operational handling". Author of the monography "Modular architecture of manufacturing machines".

Peter Demeč, prof. Ing. CSc., (born in 1952), professor in Production machines and equipment (since 2003) at the Faculty of Mechanical Engineering TU in Košice (SjF TUKE). Graduated SjF TUKE in 1975, scientific degree Candidate of Sciences (CSc./PhD.) defended at the Slovak Technical University in Bratislava (1984), to associate professor habilitated on SjF TUKE in 1994. Vice dean of SjF TUKE (1993-1996), Head of the Department of Manufacturing Technology (1997-2002), Deputy Head of the Department of Manufacturing Technology and Robotics (2003-2014), Head of Department of Manufacturing Technology (2014- present). Member of Scientific council SjF TUKE, Faculty of Environmental and Manufacturing Technology University in Zvolen, Faculty of Special Technology and University of Alexander Dubček in Trenčín, a member of the Society for 
Acta Mechanica Slovaca

Journal published by Faculty of Mechanical Engineering - Technical University of Košice

machine tools the Czech Republic, Club President of the Slovak Association of Mechanical Engineers for SjF TUKE, Member of Editorial Board of MM Science Journal. Research interests of machine tools construction, virtual prototyping, and precision machine tools. Author of the monograph "The accuracy of machine tools and its mathematical modelling" (Košice: Vienala 2001), head author of the monograph "Virtual prototyping of the machine tools in terms of the dynamics of the machining process "(Košice: SjF TUKE 2011) and over 160 scientific and professional articles in journals and contributions to scientific and professional conferences.

Luboslava Šidlovská, Ing., (born in 1965) she graduated in Automated machinery at Technical University of Košice in 1988. She was an assistant at the Department of Industrial Robotics in Prešov. Nowadays she works as a researcher for the Department of Production Technique at the Faculty of Mechanical Engineering at Technical University of Košice. She is an author, co-autor for more then 55 publications in journals and conference publications in Slovakia and abroad. She has worked on several grant projects, research tasks and projects solutions for industry that were realized within the department. 